\title{
Daniel Gouadec, Profession:traducteur
}

\section{Maria Margherita Mattioda}

\section{Q OpenEdition}

\section{Journals}

\section{Edizione digitale}

URL: http://journals.openedition.org/studifrancesi/33977

DOI: 10.4000/studifrancesi.33977

ISSN: 2421-5856

\section{Editore}

Rosenberg \& Sellier

\section{Edizione cartacea}

Data di pubblicazione: 1 décembre 2005

Paginazione: 700

ISSN: 0039-2944

\section{Notizia bibliografica digitale}

Maria Margherita Mattioda, «Daniel Gouadec, Profession:traducteur», Studi Francesi [Online], 147 (XLX |

III) | 2005, online dal 30 novembre 2015, consultato il 18 avril 2021. URL: http://

journals.openedition.org/studifrancesi/33977 ; DOI: https://doi.org/10.4000/studifrancesi.33977

\section{Questo documento è stato generato automaticamente il 18 avril 2021.}

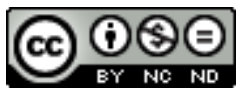

Studi Francesi è distribuita con Licenza Creative Commons Attribuzione - Non commerciale - Non opere derivate 4.0 Internazionale. 


\title{
Daniel Gouadec, Profession:traducteur
}

\author{
Maria Margherita Mattioda
}

\section{NOTIZIA}

DANIEL GOUADEC, Profession:traducteur, Paris, La Maison du Dictionnaire, 2002, 432 pp.

http://www.profession-traducteur.net/index.htm

1 La figura del traduttore è apparsa spesso ambigua, sfuggente e difficilmente inscrivibile in un quadro professionale di arti e mestieri in quanto troppo eclettica, dinamica e flessibile. Sebbene sia ormai solidamente fondata la traduttologia e copiosi siano gli studi teorici relativi alla traduzione, più limitate sono le riflessioni sul rapporto tra la natura della traduzione e il suo esercizio da parte degli "addetti ai lavori", ovvero dei traduttori. Daniel Gouadec, specialista in traduzione e terminologia dell'Université di Rennes 2, si avventura nel volume Profession: traducteur sulla via della definizione del traduttore in quanto figura professionale del XXI secolo altamente qualificata e dotata delle competenze necessarie a seguire le evoluzioni della società che richiede le sue prestazioni. La "fisiologia" del traduttore, che già il XIX secolo aveva tentato di abbozzare, diviene oggetto di un'indagine che intende offrire un quadro generale delle professionalità, dei mercati, degli operatori implicati nella traduzione, attraverso l'osservazione dei mutamenti sopraggiunti negli ultimi dieci anni che ne hanno radicalmente modificato le condizioni, i processi, le procedure e il contesto. In sostanza, si sottolinea la transizione da una tipologia di traduttori vagamente definiti ad un nuovo modello di traduttore altamente specializzato che il sottotitolo del volume identifica come "Alias Ingénieur en communication multilingue (et) multimedia", proprio ad indicare il profilo tecnologico a cui esso tende: "ce personnage qui connait les cultures et les langues confrontées dans la traduction, qui connaît les domaines de spécialité ou d'expérience auxquels renvoient les documents à traduire, qui maîtrise les techniques, stratégies, et enjeux de la traduction ainsi que tous les outils permettant de gérer les matériaux les plus divers [...]: le traducteur+ rédacteur+quasi-informaticien+bidouilleur 
de génie (mâtiné de terminologue-phraséologue et doublé d'un bon gestionnaire“ (p. 375).

2 Il saggio, ripercorrendo linee già tracciate in precedenti volumi dall'autore, si articola in sei sezioni volte ad illustrare la natura della traduzione (L'univers $d u$ traducteurL'univers de la traduction); i suoi mercati, regolati economicamente dalla domanda e dall'offerta (Les marchés); l'organizzazione del lavoro del traduttore, le sue prestazioni e la sua deontologia (La traduction au quotidien); il contesto socio-economico e culturale in cui si svolge l'attività traduttiva (Dans l'œeil du cyclone - Chronique de tempêtes passées, présentes et à venir); i profili delle competenze richieste (Devenir traducteur) e gli interrogativi sui percorsi formativi più consoni alla costruzione delle professionalità in ambito traduttivo (Le problème (?) de la formation).

Daniel Gouadec si propone di offrire, mediante un approccio analitico al mestiere, un vero e proprio stato dell'arte sull'universo della traduzione professionale - sia essa letteraria, tecnico-scientifica o multimediale -, intesa come attività esercitata quotidianamente da "une entité qui se singularise par des variables [...]" (p. 31). Particolare attenzione viene riservata ai problemi che interessano il mondo dei traduttori come l'informatizzazione degli ambienti di traduzione (TAO), la globalizzazione dei mercati, l'internazionalizzazione, le condizioni di lavoro e gli obiettivi di produttività, l'industrializzazione dei processi e degli strumenti, la diversificazione dei materiali da tradurre e la specializzazione, l'evoluzione dei profili delle competenze, ecc., al fine di fornire risposte adeguate a coloro che operano nel settore o che intendono intraprendere questa attività, oltrechè per tentare di delineare il futuro del mestiere. L'opera è destinata, infatti, ai traduttori che intendono aggiornarsi costantemente sui cambiamenti in atto nella professione, agli aspiranti traduttori, ai docenti che devono orientare gli studenti e informarli sulle formazioni in traduzione, ai formatori di traduttori e, in generale, a tutti coloro che vogliono conoscere meglio una professione "étonnament méconnue - sans doute parce que tout le monde croit, bien à tort, savoir ce que traduire veut dire".

Segnaliamo, in appendice, l'elenco delle formazioni per traduttori esistenti nelle Università francesi, la lista delle associazioni professionali, con relativi indirizzi Internet, e un glossario di termini utilizzati che rendono il volume una valida guida per inoltrarsi nel settore della traduzione. 